\title{
Rethinking the kelvin
}

\author{
Michael de Podesta discusses the current definition of the kelvin — and why it is worth changing one last time.
}

T here is a limit to the smallest conceivable uncertainty in any measurement: no quantity can be measured more accurately than the unit of that quantity can be defined. With this in mind, metrologists plan to redefine four of the seven SI base units in 2018, essentially removing uncertainty from the unit definitions and placing it all in the measurement domain. How will this redefinition affect the kelvin ${ }^{1}$ ?

Measurement is the quantitative comparison of an unknown quantity with a standard. Temperature standards are based on naturally occurring 'fixed-points' - typically, the melting or freezing points of pure substances. Thermometric pioneers typically picked two fixed-points and a thermometric indicator - such as the expansion of a liquid in a glass capillary - and then asserted that the indicator varied linearly with temperature between the fixed-points. This allowed reproducible measurements but provided no escape from the 'catch-22' of thermometry: the linear response of a thermometer cannot be verified unless it is checked with... another thermometer! In the mid-19th century, the air thermometer based on the ideal gas law came to be considered the most trustworthy and formed the basis of the earliest reliable estimates of the relative temperatures of fixed points ${ }^{2}$.

From these early roots, thermometry has grown as two interwoven branches practical and fundamental. Improvements in practical thermometry led to progress in science and technology as chemical and technological processes could be first studied, and then optimized. And improvements in fundamental thermometry and thermodynamics led to insights about the nature of temperature. Importantly, as our understanding developed, it became possible to construct primary thermometers that enabled us to escape from the catch-22 by determining the ratio of the temperatures of, say, the freezing points of sulfur and of tin, from first-principles physics.

Throughout the 20th century, the Consultative Committee for Thermometry of the Bureau International des
Poids et Mesures oversaw the production of a series of practical temperature scales based on our increasingly accurate knowledge of the temperatures of a range of fixed-points. Importantly, in 1948 the degree Celsius linked to the kelvin - was introduced and the term degree Centigrade dropped because the temperature scale was no longer based on there being 100 degrees between the boiling and freezing points of water. However the link between thermometry and the properties of 'pure' water was retained when, in 1954, the temperature of the triplepoint of pure water, $T_{\text {TPW }}$, was adopted as our temperature standard and defined to be 273.16 K exactly. As a consequence, since then, every temperature measurement has been indirectly a comparison against this standard temperature.

The choice of $T_{\text {TPW }}$ was clever: a sealed glass cell containing pure water is not difficult to make, and yields temperature measurements traceable to a universal physical phenomenon. Additionally, $T_{\mathrm{TPW}}$ is remarkably reproducible: in a recent intercomparison, cells from around the world varied by typically $30 \mu \mathrm{K}$ (ref. 3 ).

However, the choice of $T_{\text {TPW }}$ as a standard has drawbacks: no temperature measurement can ever be more accurate than the uncertainty with which $T_{\text {TPW }}$ can be realized. And comparisons of thermometric quantities inevitably become less accurate at temperature extremes. Moreover, despite

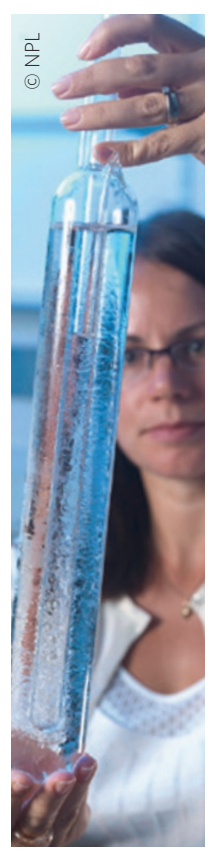
what we know about the physical meaning of temperature, the definition of the kelvin is not linked to any concept of energy.

The planned 2018 redefinition will follow the example of other SI units and establish an abstract definition with no intrinsic uncertainty that should not require alteration even in the face of centuries of unforeseeable technological change $e^{4}$. Instead of choosing an arbitrary temperature as an exact standard, we will instead state an exact value of the Boltzmann constant, $k_{\mathrm{B}}$. This specifies the amount of energy (in joules) per (statistical-mechanical) degree of freedom that corresponds to one kelvin.

To choose the value to be fixed, my own team ${ }^{4}$ and others ${ }^{5}$ have made measurements of the product $k_{\mathrm{B}} T_{\mathrm{TPW}}$ using a range of physical principles ${ }^{6}$. From 2018 onwards, the implied value of $k_{\mathrm{B}}$ in the current system will become a defined value in the new system with no uncertainty. And from then on, any measurement of temperature will be fundamentally a measure of the level of molecular kinetic energy measured in joules - and hence traceable to the fundamental constants $h$ and $c$.

The redefinition won't make measuring temperature easier. However, it will lift limits on potential future improvements in measurement techniques. For example, using a fixed value of $k_{\mathrm{B}}$ in the Nyquist formula for noise thermometry at cryogenic temperatures, or in the Planck radiation law at high temperatures, we can deduce temperature without the need to refer to $T_{\text {TPW. }}$. But personally, I consider the most compelling reason for the redefinition is this: if we had collectively understood what temperature was when we learned to measure it, then we would have defined the unit of temperature in this way in the first place. If a thing is worth doing, it is worth doing late. $\square$

\section{MICHAEL DE PODESTA is at the}

Engineering Measurements Division of the National Physical Laboratory, Teddington, TW11 OLW, UK.

e-mail: michael.depodesta@npl.co.uk

\footnotetext{
References

1. Fischer, J. \& Ullrich, J. Nature Phys. 12, 4-7 (2016).

2. Chang, H. Inventing Temperature (Oxford Univ. Press, 2004)

3. http://go.nature.com/ $6 \mathrm{MqiJK}$

4. de Podesta, M. et al. A low-uncertainty measurement of the Boltzmann constant. Metrologia 50, 354-376 (2013).

5. Fischer, J. et al. Temperature: Its Measurement and Control in Science and Industry Vol. 8, 1-10 (AIP, 2013).

6. Moldover, M. R., Tew, W. L. \& Yoon, H. W. Nature Phys. 12, 7-11 (2016).
}

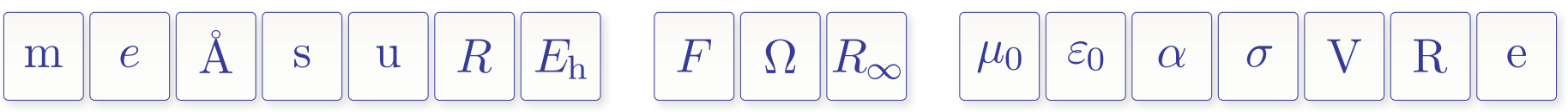

